\title{
Circumnuclear Dynamics in Mrk 273 and Mrk 231
}

\author{
A.M.S. Richards, R.J. Cohen, G.H. Cole, A.J. Holloway, A. Pedlar \\ JBO, University of Manchester, Cheshire, SK11 9DL, UK.
}

J.L. Collett, J.H. Knapen, J.A. Yates

Dept. of Physical Sciences, University of Hertfordshire, AL10 9AB, UK.

D. Field

Institute of Physics and Astronomy, University of Aarhus, Denmark.

M.D. Gray

Dept. of Physics, UMIST, PO Box 88, Manchester M60 1QD, UK.

C.G. Mundell

ARI, Liverpool John Moores University, Birkenhead, CH41 1LD, UK.

M.M. Wright

School of Chemistry, University of Bristol, Bristol, BS8 1TS, UK.

\begin{abstract}
Markarian 273 and Markarian 231 are ultra-luminous IR galaxies which show signs of merger activity. Their radio continuum and spectral line emission has been imaged on scales of tens of parsec using MERLIN. Mrk 273 and Mrk 231 are classed as Seyfert types 2 and 1 respectively. The distribution and velocity gradients shown by $\mathrm{OH}$ masers and $\mathrm{HI}$ absorption are consistent with material in a rotating disk or ring, with the axis at greater than $45^{\circ}$ to the line of sight in Mrk 273, and at less than $45^{\circ}$ to the line of sight in Mrk 231. We estimate the amount of mass enclosed and infer its distribution from irregularities in the maser morphologies and comparison with $\mathrm{HI}$ and radio continuum data.
\end{abstract}

\section{Introduction}

Mrk 273 has a systemic velocity of $V_{\mathrm{LSR}}=10837 \mathrm{~km} \mathrm{~s}^{-1}$ in the radio convention, and a redshift of $z=0.038$. Mrk 231 is at $V_{\mathrm{LSR}}=12137 \mathrm{~km} \mathrm{~s}^{-1}, z=0.042$. Both are very bright IRAS galaxies, with far infra-red luminosities $\log \left(L_{\mathrm{FIR}} / \mathrm{L}_{\odot}\right)$ of 12.48 and 12.04 respectively. Mrk 273 has an $\mathrm{HI}$ absorption column density of $17-18 \times 10^{23} \mathrm{~m}^{-2} \times$ (spin temperature/filling factor), almost three times greater than the density towards Mrk 231 (Cole et al. 1999; Carilli Wrobel \& Ulvestad 1998), consistent with their Seyfert characteristics. 


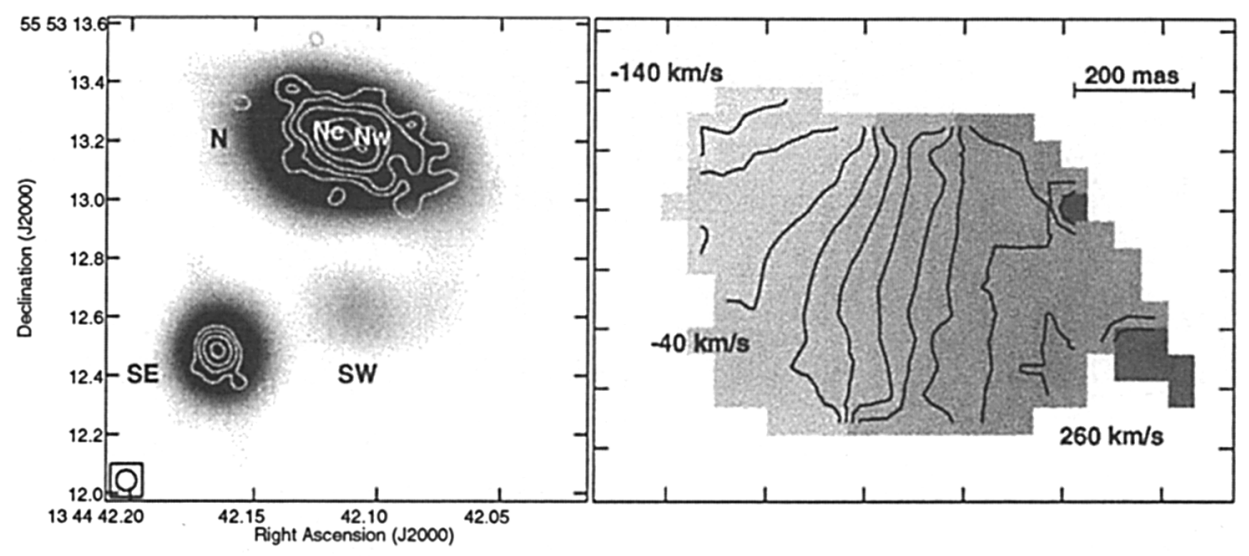

Figure 1. Mrk $273(l)$ Radio continuum at $1.6 \mathrm{GHz}$ (greyscale) and $5 \mathrm{GHz}$ (contours, $1,2,4 \ldots \times 0.3 \mathrm{mJy} \mathrm{bm}^{-1}$ ) (Knapen et al. 1997). $(r)$ HI absorption towards region $N$ (Cole et al. 1999), velocities are with respect to the systemic velocity of Mrk 273.

Optical images of Mrk 273 show a dramatic tidal tail and multiple nuclei (Borne et al. 1999), and it appears to have undergone mergers within the last $10^{8} \mathrm{yr}$. Comparison of 1.6 and $5 \mathrm{GHz}$ radio continuum images and IR data (Knapen et al. 1997) show the northern nucleus of Mrk 273 has twin peaks (Fig. $1(l) \mathrm{Ne}, \mathrm{Nw}$ ) with flat radio spectra, probably associated with the Seyfert nucleus/nuclei although VLBI imaging (Carilli \& Taylor 2000) suggests $\mathrm{Ne}$ is itself multiple and there is evidence for starburst activity. Mrk 231 also has a disturbed history on a timescale of $10^{9} \mathrm{yr}$ (Soifer et al. 2000). Ulvestad et al. (1997) found VLBI-scale jets roughly north-south, approaching to the south.

\section{Observations}

Red-shifted $\mathrm{OH}$ mainline maser emission from Mrk 273 (Yates et al. 2000) and Mrk 231 was observed in 1997 using MERLIN ${ }^{1}$ with a channel width of 22.5 $\mathrm{km} \mathrm{s}^{-1}$ and a beamsize of $100-200$ mas depending on weighting. After continuum subtraction maser feature properties were measured by fitting Gaussian components with a relative positional accuracy of $2-28$ mas, in addition to the absolute position uncertainty of $\sim 10$ mas. Our results are compared with HI absorption mapping of Mrk 273 (Cole et al. 1999) shown in Fig. 1(r).

\section{Results}

The distributions of maser components are shown in Fig. 2. In most places 1665and (brighter) $1667-\mathrm{MHz}$ masers are seen at similar velocities. In Mrk $273(l)$

\footnotetext{
${ }^{1}$ MERLIN is the UK national radio astronomy facility operated by the University of Manchester at Jodrell Bank Observatory on behalf of PPARC
} 

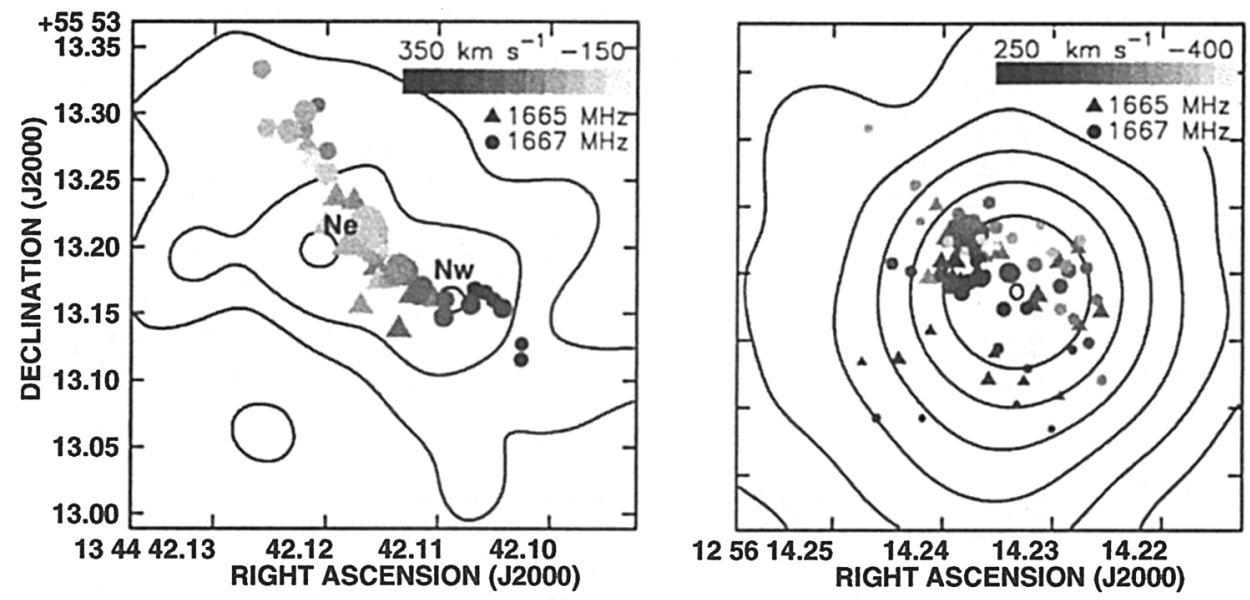

Figure 2. $\mathrm{OH}$ masers (symbol area $\propto$ flux density) superimposed on $1.6 \mathrm{GHz}$ continuum contours (at $1,2,4 \ldots \times 1.9 \mathrm{mJy} \mathrm{bm}^{-1}$ ) in $(l)$ Mrk 273 and $(r)$ Mrk 231. Velocities are relative to the systemic velocity.

we detected emission over a $V_{\text {LSR }}$ range of $500 \mathrm{~km} \mathrm{~s}^{-1}$. The elongated emitting region forms two clumps, each containing both maser lines. The brighter clump straddles $\mathrm{Ne}$ and $\mathrm{Nw}$ and has a clear position-velocity gradient suggesting a rotating disc almost perpendicular to the plane of the sky. The maser emission from Mrk 231 has a $V_{\mathrm{LSR}}$ extent of $700 \mathrm{~km} \mathrm{~s}^{-1}$ containing five clumps of emission from the $1667-\mathrm{MHz}$ line, four of which have corresponding $1665-\mathrm{MHz}$ emission. The masers occur in a roughly circular region which could be a disc lying close to the plane of the sky, slightly tilted with the sense of rotation such that material is receding in the SE and approaching in the NW. This orientation is also consistent with the faintness of the Mrk 231 masers $\left(\leq 26 \mathrm{mJy} \mathrm{bm}^{-1}\right)$ compared with those in the more nearly edge-on disc in Mrk $273\left(\leq 67 \mathrm{mJy} \mathrm{bm}^{-1}\right)$. In contrast the peak 1.6-GHz continuum emission from the maser region of Mrk 231 is $146 \mathrm{mJy}$ $\mathrm{bm}^{-1}$, eight times brighter than that from Mrk 273.

If the masing disc is in either Keplerian or solid body rotation, the enclosed mass density is given by $\rho_{\mathrm{M}} \approx(V / r)^{2} /[(4 / 3) \pi \times 0.0043] \mathrm{M}_{\odot} \mathrm{pc}^{-3} \cdot r$ is the half-width of the masing disc in pc (using $H_{0}=75 \mathrm{~km} \mathrm{~s}^{-1} \mathrm{Mpc}^{-1}$ ). The change in $V_{\mathrm{LSR}}$ across the disc (tilted at $i^{\circ}$ to the plane of the sky) is $2 V \sin i$ where $V$ is the rotation velocity of the disc at $r$. This is also used to find total mass enclosed, $M$, which is a lower limit if only part of the disc is detected. Table 1. compares the estimates derived from $\mathrm{OH}$ maser observations with $\mathrm{HI}$ results.

The biggest uncertainties arise from likely deviations from smooth rotation, for example clutches of $\mathrm{OH}$ masers in Mrk 273 with anomalous velocities suggest either multiple discrete mass concentrations or infall as well as rotation (Yates et al. 2000), and there is evidence of infall in some $\mathrm{HI}$ absorbing regions in both galaxies (Carilli et al. 1998, Carilli \& Taylor 2000). It is almost certain that for Mrk 273, $i>45^{\circ}$, and for Mrk 231, $i<45^{\circ}$. We adopt intermediate values of $i=67.5^{\circ}$ and $i=22.5^{\circ}$ respectively which could produce an error of up to $70 \%$ for Mrk 273, and several $100 \%$ for Mrk 231. Nonetheless two results 
stand out from the maser data. Firstly, within $90 \mathrm{pc}$ of the core of Mrk 231, $M \approx 6 \times 10^{9} \mathrm{M}_{\odot}$, ten times greater than the mass within $50 \mathrm{pc}$ of the core of Mrk 273. Secondly, within Mrk 273 the agreement between the two HI results on different scales and the contrast with the $\mathrm{OH}$ result suggests that $\rho_{\mathrm{M}} \sim 230$ $\mathrm{M}_{\odot} \mathrm{pc}^{-1}$ at a few $100 \mathrm{pc}$ from the nucleus, but within the central $50 \mathrm{pc}$ there is an extra $\sim 0.5 \times 10^{8} \mathrm{M}_{\odot}$.

Table 1. The mass density in the nuclear regions of Mrk 231 and Mrk 273 from $\mathrm{OH}$ and $\mathrm{HI}$ velocity gradients.

\begin{tabular}{l|rr|rrr}
\hline & \multicolumn{3}{|l|}{ Mrk 231 } & \multicolumn{3}{l}{ Mrk 273 } & \\
& HI (1) & OH (2) & OH (3) & HI (4) & HI (5) \\
\hline$r(\mathrm{pc})$ & 70 & 90 & 50 & 110 & 200 \\
$V \sin i\left(\mathrm{~km} \mathrm{~s}^{-1}\right)$ & 110 & 210 & 220 & 225 & 400 \\
$\rho_{\mathrm{M}} \sin ^{2} i\left(\mathrm{M}_{\odot} \mathrm{pc}^{-1}\right)$ & 140 & 300 & 1080 & 230 & 220 \\
$M \sin ^{2} i\left(10^{9} \mathrm{M}_{\odot}\right)$ & 0.2 & 0.9 & 0.6 & 1.3 & 7.4 \\
\hline \hline
\end{tabular}

(4) Carilli \& Taylor 2000 (5) Cole et al. 1999

\section{Conclusions}

Mrk 231 and Mrk 273 have many similarities, but differ notably in their Seyfert type, and it it likely to be longer since Mrk 231 underwent a merger. As well as confirming the presence and orientation of a molecular disc on the scale of tens of pc, these $\mathrm{OH}$ maser observations reveal the kinematics and the enclosed mass density. This can be used to test the suggestion first elaborated by Sanders et al. (1988) that ULIRGs originate in mergers and evolve through starburst and AGN phases, as Mrk 273 shows more pronounced multiple nuclei and starburst activity whilst Mrk 231 has a larger central mass and jet activity.

\section{References}

Borne K. D., Bushouse, H., Colina, L., Lucas, R. A., Baker, A., Clements, D., Lawrence, A., Oliver, S., Rowan-Robinson, M. 1999, Ap\&SS, 266, 137

Carilli, C. L., Wrobel, J. M., Ulvestad, J. S. 1998, AJ, 115, 928

Carilli, C. L., Taylor, G. B. 2000, ApJ, 532, L95

Cole, G. H. J., Pedlar, A., Holloway, A. J., Mundell, C. G. 1999, MNRAS, 310, 1033

Knapen, J. H., Laine, S., Yates, J. A., Robinson, A., Richards, A. M. S., Doyon, R., Nadeau, D. 1997, ApJ, 490, L29

Sanders, D. B., Soifer, B. T., E,ias, J. H., Madore, B. F., Matthews, K., Neugebaguer, G., Scoville, N. Z. 1988, ApJ, 325, 74

Yates, J. A., Richards, A. M. S., Wright, M. M., Coillett, J. L., Gray, M. D., Field, D., Cohen, R. J. 2000, MNRAS, 317, 28 